\title{
Anesthetic management in laparoscopic living donor hepatectomy, the first case-series in Thailand
}

\author{
Phuriphong Chanthima ${ }^{1}$, Warangkana Lapisatepun ${ }^{1}$, Atipa Nitayamekin ${ }^{1}$, Suraphong Lorsomradee ${ }^{1}$, Settapong Boonsri ${ }^{1}$, \\ Worakitti Lapisatepun ${ }^{2}$, Sunhawit Junrungsee ${ }^{2}$, Anon Chotirosniramit ${ }^{2}$
}

${ }^{1}$ Department of Anesthesiology, Faculty of Medicine, Chiang Mai University, Chiang Mai, Thailand
${ }^{2}$ Department of Surgery, Faculty of Medicine, Chiang Mai University, Chiang Mai, Thailand

Background: Laparoscopic living donor hepatectomy began early in 2020 at our institution. This surgery was the first case and a new experience in Thailand. Perioperative and anesthetic management should not only concern anesthetic and surgical complications but also graft function, including ischemic time. In this paper, we review anesthetic considerations and perioperative management in laparoscopic living donor hepatectomy as a new experience in Thailand.

Methods: All four living donors were selected and underwent elective laparoscopic living donor hepatectomy at the university hospital.

Results: Differences in anesthetic considerations between laparoscopic liver resection in pathologic livers versus donor liver graft typically include heparin administration, intravenous indocyanine green, and prolong ischemic time after major vessels clamping. During the operation, only one case had subcutaneous emphysema and one other case had oliguria. There were no intraoperative hypothermia in all cases. All the patients were able to be extubated at the end of surgery inside the operation room. For postoperative pain control, two cases were given intravenous morphine patient-controlled analgesia, while the others were given continuous intravenous morphine infusion. However, emergent explore laparotomy was performed in one case to stop postoperative bleeding from the surgical site.

Conclusions: The advantages of minimal invasive technique include a potential reduction in blood loss and transfusion requirement, lower incidence of intraoperative hypothermia, as well as decreased postoperative pain and opioid consumption. However, complications that must be taken into account include the effect of pneumoperitoneum, patient's positioning, and emergent conversion to open surgery. As this surgery is largely dependent on the surgeon's experience, operation time may be extended in some cases. As an anesthesiologist, practical and effective anesthetic management is essential to improve patient outcomes.

Corresponding author: Warangkana Lapisatepun

E-mail: warangkana.c@cmu.ac.th

\section{(c) The Korean Society for Transplantation}

This is an Open Access article distributed under the terms of the Creative Commons Attribution Non-Commercial License (http://creativecommons.org/licenses/by-nc/4.0/) which permits unrestricted non-commercial use, distribution, and reproduction in any medium, provided the original work is properly cited. 